\title{
Mobile Agent Initiated Energy Efficient Data Aggregation in WSNs: MAEDA
}

\author{
Shivangi Katiyar \\ Department of Computer Science and \\ Engineering, \\ M.M.University, Solan, Himachal Pradesh, India
}

\author{
Devendra Prasad \\ Dr. Devendra Prasad Prof. (CSE Department) \\ Chandigarh Engineering College, Landran, \\ Mohali, Chandigarh
}

\begin{abstract}
A sensor node in WSNs has limited resources (e.g., memory capacity, battery power etc.) and among all, energy is the most crucial factor because refilling or recharging of power is not possible here. There are many possible ways to conserve energy in WSNs; data aggregation is one of them. Applications of WSNs cross a broad scale including environment monitoring, Habitat monitoring, monitoring Water and Air quality, Gas emission, etc. The above mentioned applications and many other needs Data Aggregation because here sensors frequently reports sensed values to the Processing Elements without removing redundant and disused entries. This paper proposes Mobile Agent initiated Energy efficient Data Aggregation (MAEDA) approach to prolong network lifetime. This method skillfully integrates Mobile Agent (MA) with wireless sensor networks. The proposed method is emulated by MATLAB platform; our simulation results are very impressive and prove that MAEDA is able to save more energy in data aggregation as well as prolonged network lifetime than other existing one.
\end{abstract}

\section{Keywords}

Sink, Mobile Agent, Data aggregation.

\section{INTRODUCTION}

Wireless sensor networks (WSNs) are fascinating both industries as well as research and bring new advancement in mobile computing and wireless communications [1]. A sensor node in WSNs has limited resources (e.g., memory capacity, battery power etc.) and among all, energy is the most crucial factor because refilling or recharging of power is not possible here [2]. Many researchers have been proposed lots of schemes for better utilization of the node's energy. There are many possible ways to conserve energy in WSNs; data aggregation is one of them. Data Aggregation involves data gathering from the surroundings as well as remove redundant data entries so that to maximize the useful content in data and reduce the volume of data. Data aggregation is very profitable where summarized information is required, in such cases some nodes aggregate data at different points of the network and finally delivers it to the sink instead of individual transfer of data to the sink [3]. Applications of WSNs cross a broad scale including environment monitoring (Water level, Forest fire, Temperature, Humidity etc.) [4], Habitat monitoring [5], monitoring Water and Air quality, Gas emission, etc. [6]. The above mentioned applications and many other needs Data Aggregation because here sensors frequently reports sensed values to the Processing Elements without removing redundant and disused entries. Here we are proposing Mobile Agent initiated Energy efficient Data Aggregation approach (MAEDA) which skillfully integrates Mobile Agent (MA) with wireless sensor networks whereas Mobile Agent is basically a software agent [7]. According to our proposed algorithm MA has to traverse the network in the form of Tree. Here sink sends mobile agent to traverse whole network for the purpose of sending parent related information to child. When all nodes become aware about their respective parent then MA dissolves at leaf nodes; Reverse tree processing starts from these end nodes. In Reverse tree processing, each child node starts sending data to their own respective parent and each parent has to aggregate data from their child nodes. Our results are very impressive, and prove that proposed method is able to save more energy than previously existing approaches and manages topology also. The rest of the paper is organized as follows. Section II presents related work. Section III introduces system model along with proposed algorithms. Section IV elaborates the results and discussions while Section V concludes the paper.

\section{MOBILE AGENTS}

MAs are the sign of intelligent exploitation of software technology; because it's all about software programs. MA is defined as an autonomous software program which runs on behalf of network user. The appeal of Mobile Agents is quite tempting now days because of their amazing features such as autonomy, social ability, learning and most appreciably mobility. In addition MAs are able to interact with outdated processing system.

In a traditional client server based computing model, an operation is split into two parts across a network i.e. request and response where clients make request from a user machine to a server which services the requests on a large, centralized system. This model is advantageous for certain applications including the removal of the client to smaller, remote machines etc. but it breaks down under other circumstances, including slow/poor quality network connections, highly distributed systems, and particularly in the situation of changing applications. Additionally in this model the client needs good network bandwidth, reliable server connection, and assurance of response.

Mobile agents overcome all these intrinsic limitations in client/server [white paper]. For the use of MAs no application level protocol is required as well as they are comfortable in distributed environment also. Most importantly an agent can compromise with network quality at some extent because it carries own credentials so connection is just a medium for it. In recent times MA has been proposed for efficient data processing in WSNs [8-11].

There are some issues related with MAs like what will be the best route for MA to traverse and other one is size of MA; as noticed from literature $[12,13]$ MA grows larger as they start to collect data from sensor nodes. The proposed model not only deals with energy efficient data aggregation but also incorporate enhanced and dispute free MA concept. MAEDA 
provides suitable path information to MA to tackle route related issue and size related problems dissolves by restrictive use of MAs.

\section{RELATED WORK}

Till now Lots of research has been done for efficient energy utilization in WSN. WSN is a data oriented as every node might generate some data and data gathering is indeed a broad subject matter in the field of WSNs. Most of the data gathering research focuses on either the routing or data gathering, and they usually depend on infrastructures such as tree, cluster, hybrid etc. [14]. In [15] author proposes an Enhanced Forward Aware Factor-Energy Balanced Routing Method (EFAF-EBRM) with key aspects that is, reduced number of messages for setting up a routing tree, efficient and reliable data aggregation and transmission. Here they proposed a new communication protocol based on forwardaware factor in order to determine next-hop node and Data Routing for In-Network aggregation (DRINA) protocol to reduce the number of transmissions and for the purpose of balancing the energy consumption and prolonging the network lifetime.

In [16] author proposes an energy-efficient in- Network data aggregation approach for WSNs. Here authors use the advantages of both cluster and tree. Basic concept which works behind this approach is that, all nodes transmit their data to their neighbor instead of their cluster head. Therefore, the communication distance is reduced and the energy consumption of each node, each cluster and the average energy consumption of the whole networks is reduced and the network lifetime is increased. Also, in the proposed approach, when the entire nodes received the information packet, each node selects it parent which should sent its data to it. The most appropriate parent according to some benchmarks will be selected for each node which can balance the network load.

In [17] authors calculate all possible routes represented by the aggregation trees with the help of genetic algorithm (GA). GA finds the optimum tree which is able to balance the data load and the energy in the network. The proposed technique would be suitable for a homogeneous WSN with some spatial correlation to monitor the surroundings. In this study, sensor nodes monitor the interested area to gather data and then remove the redundant ones in order to aggregate them according to the data aggregation spanning tree. Just then, they send packet data to the appropriate neighbors to conduct the packets toward to BS through a route which consumes low energy. BS is a powerful station which all sensed data is gathered by sensor nodes and will be collected in here in order to process special goals.

Traditional WSNs technology assumed dense network so there were lots of option for data gathering and routing but due to the advancement of technology and increasing demand of applications, technology entered into new phase that is distributed networking. So recently there is a research fashion that adopts mobile elements for the data transmission and gathering in wireless sensor networks [18, 19].

In [20] authors propose a hybrid data collection scheme called Hybrid Agent Tree Scheduling (HATS) for making flexible task execution in WSN. HATS leverages the broadcast nature of wireless transmission, the flexibility of mobile agents, and the efficiency of tree-based data aggregation to provide a solution which is as adaptive as traditional agent systems and captures the efficiency of traditional tree-based aggregation systems. This method hybridizes agent-based task dissemination and tree-based aggregation. HATS also inherently address the need for multiple itineraries by embedding those itineraries in the tree branches. Itineraries of Mobile agents, which are a useful abstraction for network retasking, were addressed from the TSP angle in previous works. This introduced the problem of grouping source nodes optimally under multiple itineraries. We, however, found that MA-dissemination using NWST approximation is more efficient. In [21] CRBCC gives a good compromise between energy consumption and delay. Chains are formed based on simulated annealing (SA) algorithm. Clusters are formed based on y co-ordinate and chain leaders are formed on x coordinates.[22] Discusses about Traditional deployments of wireless sensor networks (WSNs) rely on static base stations for collecting data. For dynamic data generation applications such as tracking and detection applications, static base stations suffer from communication bottlenecks, which cause reliability and lifetime hurdles. For addressing such type of problems, they give holistic solution where the synergy of the WSN and the mobile base station improves the reliability and lifetime of data collection. The WSN component of present solution is a trivial dynamic routing tree maintenance protocol which tracks the location of the base station to provide an always connected network. The mobile base station component complements the dynamic tree reconfiguration protocol by trailing towards the data generation, and hence, reducing the number of hops the data needs to travel to the base station. While both protocols are simple and light weight, combined they lead to significant improvements in the reliability and lifetime of data collection. They also provide an analytical discussion of proposed solution along with extensive simulations. Although this focused on the data collection problem, here data spider framework readily applies also to the pursuer-evader tracking problem by treating the ROI as the evader and the $\mathrm{MB}$ as the pursuer. Experiments showed that the trail-flow algorithm for the MB manages to implicitly route the MB around the holes, a desirable property for pursuer-evader tracking.

\section{SYSTEM MODEL}

The network of the proposed model consists of large number of sensor nodes deployed randomly in the area to be monitored and a Base Station or sink placed at specified location as depicted in Fig.1 (a). The network also consists of Mobile Agent (MA), which is a software agent and perform the action directed by sink. It is assumed that the network is location aware i.e. location information of all nodes are known and maintained at Base station or Sink and first order radio model [23] is used for the computation of energy consumption in data transmission by sensor and is given below.

$E_{t x}(k, d)=E_{t x}-\operatorname{elec}(k)+E_{t x}-\operatorname{amp}(k, d) \ldots \ldots \ldots$
$E_{r x}(k, d)=E_{r x}-\operatorname{elec}(k) \ldots \ldots \ldots \ldots \ldots \ldots \ldots \ldots \ldots \ldots \ldots \ldots \ldots \ldots \ldots \ldots \ldots \ldots \ldots \ldots \ldots \ldots \ldots \ldots \ldots \ldots \ldots \ldots \ldots \ldots \ldots \ldots$

According to first order radio model Eelec is energy consumption to run the transmitter or receiver circuitry, Eamp is energy required by transmitter amplifier, $E_{D A}$ is the energy required to aggregate the data received by the child nodes, $\mathrm{k}$ is the number of bits transmitted and $d$ is the distance. Each sensor compresses the received data using data aggregation $(D A)$ techniques by a factor of 0.6 using distributed compressive sampling. 


\section{PROPOSED ALGORITHM}

\subsection{Network Formation}

The main motivation behind Network formation algorithms is to provide a pathway to MA. To setup a network; proposed scheme defines a formation strategy that forms network and that is coordinated by sink. There are a few preliminaries that sink need to take care of before it starts network formation.

(a) $E(v)>0$; for all participating node i.e. energy of every participating node must be greater than 0 .

(b) Number of participating node $\leq$ alive nodes i.e. number of participating node should not be greater than total number of alive nodes. It could be less than or equal to total alive nodes.

The situation when participants are equal to alive nodes is justified but here this is also possible that number of participant could be less than alive nodes. Because node which is alive but not in range of any node could not be considered as participating node, as this model tried to become more realistic so works with range of node. The possible situation is when number of participant is greater than total alive node; it could be possible only when any intruder nodes are there. The above said check will take care of network's security. The sink is the primary controller of the network and it is responsible for initiating network formation. Nodes that are one hop away from sink are supposed to be connected in accordance to setup parent child relationship. For further processing, rules remain same i.e. every child are supposed to become a parent and has to assumed connections with their children. The developments of these relationship remains continue until all nodes of the network have not been covered. In Algorithm 1, we report the pseudo-code of the network formation and discussed below.

\section{Algorithm 1: Network Formation}

Input:

\section{$V$, L: a sensor network $V$ with $L$ links}

\section{Notations:}

$v_{i:}$ a node of $V$

$n b r\left(v_{i}\right):$ neighbor nodes of $v_{i}$

$r$ : communication range of each node $v_{i}$

BS: base station or sink

$E \leftarrow$ a matrix

Output:

\section{$N$ : Network}

\section{Working steps:}

1. parent $=B S$

2. dist $\leftarrow$ distance from parent node to other nodes

3. for each $v_{i}$ in $V$

a. $\quad$ if $($ dist $<r)$

b. $\quad E($ row, 1$)=$ parent;

c. $E($ row, 2$)=n b r$ (parent $)$;

d. $\quad E($ row, 3$)=\operatorname{dist}($ nbr $($ parent $)) \leftarrow$ i.e. distance between parent to nbr(parent);

e. $\quad$ row $=$ row +1

f. endif

4. end

5. $V=V-n b r($ parent $)$;
6. $\quad$ parent $=n b r($ parent $) ; \leftarrow$ i.e. now all $v_{i}$ of

E(row,2) become parent one by one.

7. for each parent

8. $\quad$ end

a. repeat step 3-6

9. if $($ nbr(parent $)) \neq$ nil

a. $\quad$ goto step 7

b. else

10. endif

\subsection{Tree Formation}

The role of MA comes into action during Tree Formation Mobile Agent can move or clone themselves [24] to desired locations in response to getting instructions from sink or changing environmental conditions. The working model exploits the cloning capabilities of MA for the purpose of generating aggregation tree. Network formation and tree formation are interrelated because model achieves aggregation tree by applying tree formation algorithm over Algorithm 1 . The Tree Formation completed in two steps i.e. MA cloning and MA dissolution, as discussed below.

(i) MA cloning: in the first step MA starts their journey from the sink node and clones itself at each child of the current node. In this phase MA not only clone it at child nodes but also delivers parent related information to them. Fig.1 (b) depicts the complete MA cloning and resultant tree. (ii) MA dissolution: Role of the MA ends at the node where the current node doesn't have any child and where MA dissolves itself, in this model such type of nodes are addressed as leaf node. Fig.1 (c) depicts the complete MA dissolution.

In Algorithm 2, we report the pseudo-code of the Tree formation and discussed below:

\section{Algorithm 2: Tree Formation}

Input: Matrix E

Output:

T: data aggregation Tree

\section{Working steps:}

A. for each $n b r$ (parent) in $E$

1. $\operatorname{Count}\left(v_{i}\right)$

2. If $\operatorname{count}\left(v_{i}\right)>1$

a. For each vi

b. Compare_dist(parent,vi)

c. $\quad$ Select $\min ($ dist)

d. Connect_min_dist

$\left(v_{i}\right.$, parent $) \leftarrow$ i.e. connect

vi to the parent having

minimum distance

e. End

3. else

4. Connect $\left(v_{i}\right.$, parent $)$

B. end

5. endif

\subsection{Data Aggregation}

Because of the dense deployment, sensor nodes have overlapping sensing regions as well as redundant data entries, so for removing these unusual data entries and ignoring overlapping regions effect; data aggregation is necessary. Here we are considering continuous observation applications as adopted in [25], where the just right aggregation is possible. In such scenario every participating node is capable 
of aggregating all the packets received from its children and by itself into a sole packet before transmitting to its parent. The scale of aggregative knowledge transmitted by each node is constant and does not rely on the scale of raw sensor readings. Fig.1 (d) depicts flow of data aggregation which shows that Data Aggregation will be held at every parent; it starts from leaf and ends at the sink or root of the tree. Here the Network transformed in the form of Tree with having some valuable qualities as discussed below.

First, in the generated tree MA dissolves at the leaf of the tree after completing their dedicated tasks which restricts MA to unusual roving over the network as well as prevents from their

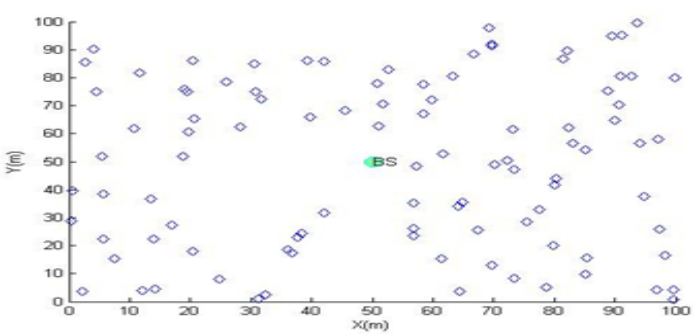

Fig. 1 (a) A Randomly deployed Sensor Network

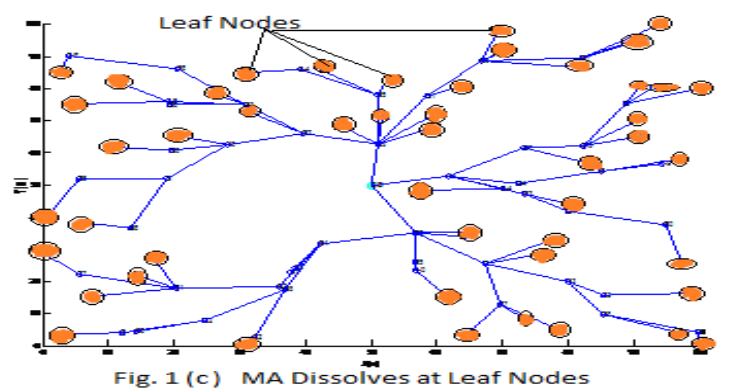

Fig 1: Data Aggregation over MA generated Tree

\section{SIMULATION RESULTS AND PERFORMANCE ANALYSIS}

The performance of MAEDA is evaluated through simulation in MATLAB, MAEDA is compared with MIEEPB (i.e., Mobile sink Improved Energy Efficient PEGASIS based) routing protocol. The network life and energy consumption is used as metrics for compares ion. The simulation is carried out for 6000 rounds and various simulation parameters are given in Table 1 below.

Table1. Simulation Parameters

\begin{tabular}{|c|c|}
\hline Parameter & Value \\
\hline Network size & $100 \mathrm{~m} * 100 \mathrm{~m}$ \\
\hline Number of nodes & 100 \\
\hline Packet size & 2000 bits \\
\hline Agent size & 200 bits \\
\hline Rounds & 6000 \\
\hline Initial energy of nodes & $0.5 \mathrm{~J}$ \\
\hline Range & $27 \mathrm{~m}$ \\
\hline $\boldsymbol{E}_{\text {elec }}$ & 50nJ/bit \\
\hline $\boldsymbol{E}_{f s}$ & 10pJ/bit/m $m^{2}$ \\
\hline$E_{m p}$ & $0.0013 \mathrm{pJ} / \mathrm{bit} / \mathrm{m}^{4}$ \\
\hline$E_{D A}$ & 5nJ/bit \\
\hline DA (Data Aggregation factor) & 0.6 \\
\hline
\end{tabular}

misuse also. Second, every node has their best parent address according to received information that is delivered by MA. Third, Data Aggregation is performed at each parent node of the tree using DCT technique as proposed in DCS [26] and final collection held at the sink node i.e. the root of the tree.
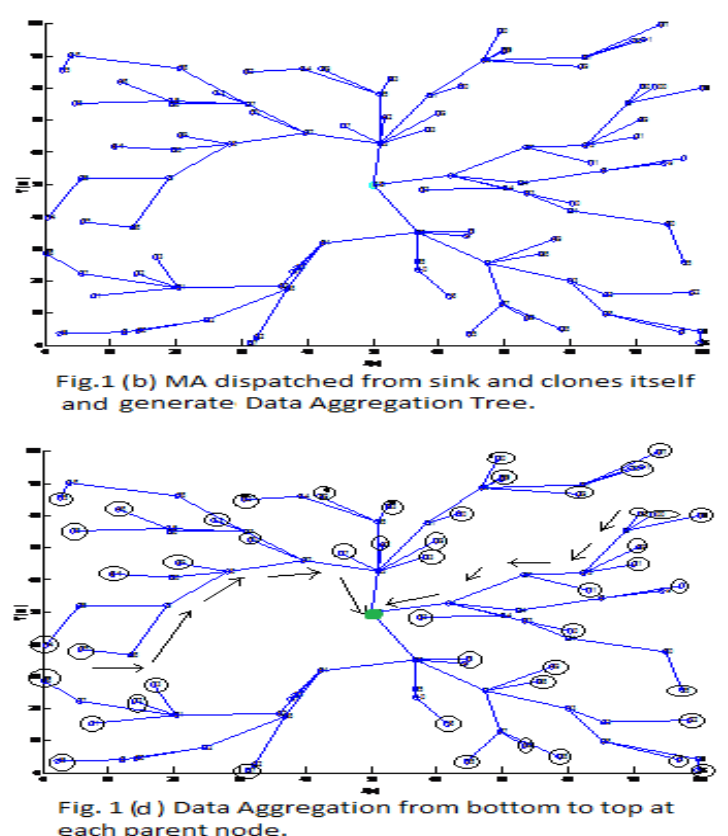

It can be observed from Fig. 2 that the permanence of MAEDA is better than MIEEPB in terms of network lifetime as in MIEEPB approximately after 4250 rounds almost all nodes are dead whereas in MAEDA about $9 \%$ nodes are still alive even after 5300 rounds which is approximately 1050 round better. Otherwise remaining nodes in MAEDA are considered as dead because they have negligible amount of energy with no network connectivity.

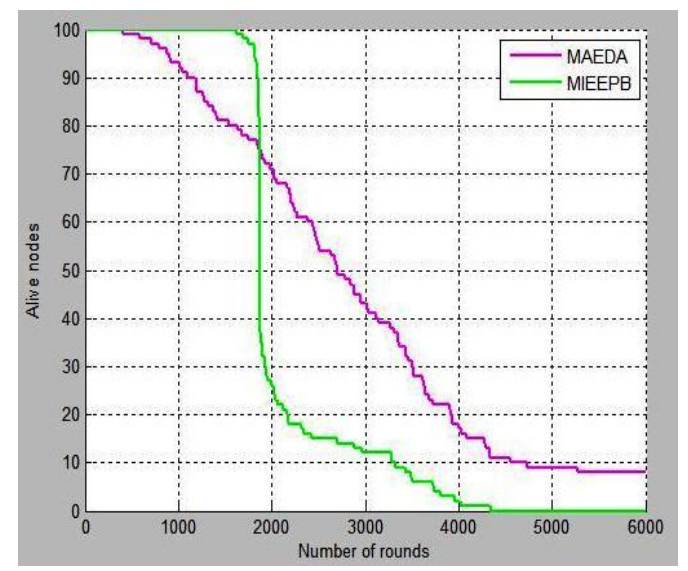

Fig. 2: Network Lifetime Graph

Fig.3. shows the instances of first dead node and the last dead node of MIEEPB and MAEDA network. It can be observed 
from fig. that in MIEEPB the number of dead nodes increases drastically after 1800 rounds and $75 \%$ nodes of the network become dead around 2000 rounds whereas MAEDA only around $30 \%$ nodes become dead in 2000 rounds and we can notice that there is no drastic increase in the numbers of dead nodes.

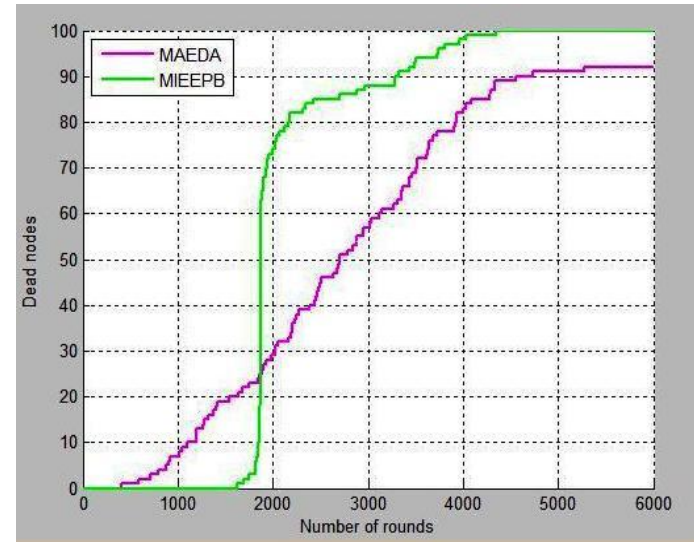

Fig. 3: Comparison of Dead nodes between MIEEPB and MAEDA

Fig.4 shows the energy consumption of MAEDA and MIEEPB protocols. In both scheme up to 4300 rounds as the number of rounds increases residual energy decreases but residual energy in MAEDA scheme is more than MIEEPB.

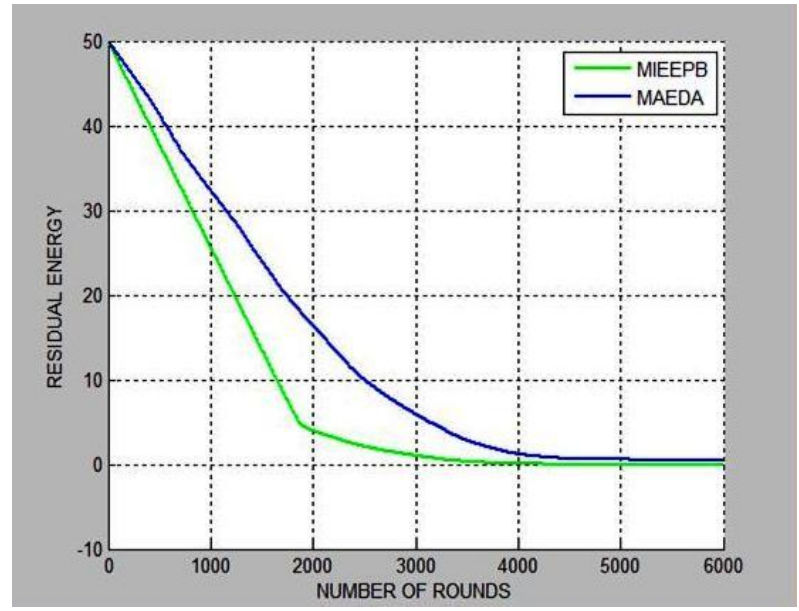

Fig. 4: Comparison of Energy consumption in MIEEPB and MAEDA

\section{CONCLUSION}

This paper proposes an energy efficient Data Aggregation approach which takes advantage of Mobile Agent (MA) to aggregate the data. In the proposed method, MA not only provides updated information to the nodes but also provides single optimized parent to each node. This fruitful information keeps only genuine link between nodes which forms a tree structure of network for further processing. It has been proved from results that integration of mobile agent (MA) with WSN has major advantage in maximizing lifetime of network.

\section{REFERENCES}

[1] Min Yoon, Yong-Ki Kim and Jae-Woo Chang, "A New Data Aggregation Scheme to Support Energy Efficiency and Privacy Preservation for Wireless Sensor Networks", International Journal of Security and Its Applications, Vol. 7, pp. 129-142, January 2013.
[2] Abdul Waheed Khan, Abdul Hanan Abdullah , Mohammad Hossein Anisi and Javed Iqbal Bangash, "A Comprehensive Study of Data Collection Schemes Using Mobile Sinks in Wireless Sensor Networks", Academic journal -Sensors, vol. 14, issue 2, pp. 2510-2548, February 2014.

[3] Md. Sajidul Islam, Imtiaz Bin Rahim and Mosarrat Jahan, "An Energy-Efficient Data Aggregation Tree Construction Algorithm for Wireless Sensor Networks", International Journal of Computer Networks and Wireless Communications (IJCNWC), Vol.4, No.5, pp.264-269, October 2014.

[4] Priya Kasirajan, "Data aggregation in wireless sensor networks", masters theses, Spring, 2010.

[5] Alan Mainwaring, Joseph Polastre, Robert Szewczyk, David Culler and John Anderson, "Wireless Sensor Networks for Habitat Monitoring", Proceedings of the 1st ACM international workshop on Wireless sensor networks and applications, WSNA '02, pp. 88-97, 2002.

[6] J. Valverde, V. Rosello, G. Mujica, J. Portilla, A. Uriarte, and T. Riesgo, "Wireless Sensor Network for Environmental Monitoring: Application in a Coffee Factory", International Journal of Distributed Sensor Networks, vol. 2012, 2012.

[7] Govind P. Gupta, Manoj Misra, and Kumkum Garg, "An Energy Efficient Distributed Approach-Based Agent Migration Scheme for Data Aggregation in Wireless Sensor Networks", Journal of Information Processing System, pp. 1-17, August 2013.

[8] Mobile agents: A new software paradigm for distributed application development, CIS-white paper.

[9] Yashpal Singh, Kamal Deep and S Niranjan, "Multiple Criteria Clustering of Mobile Agents in WSN", International Journal of Wireless \& Mobile Networks (IJWMN) Vol. 4, pp.183-193, June 2012.

[10] Ankit jagga, Kuldeep and Vijay rana, "A Hybrid Approach for Deploying Mobile Agents in Wireless Sensor Network", in proceedings of International Conference on Recent Advances and Future Trends in Information Technology (iRAFIT2012), pp.1-4, 2012

[11] Y.-C. Tseng, S.-P. Kuo, H.-W. Lee, and C.-F. Huang, "Location tracking in a wireless sensor network by mobile agents and its data fusion strategies," Computer Journal, vol. 47, pp. 448-460, 2004.

[12] H. Qi, Y. Xu, and X. Wang, "Mobile-agent-based collaborative signal and information processing in sensor networks," Proceedings of the IEEE, vol. 91, pp. 11721183, 2003.

[13] M. Rubinstein, "Scalability of mobile agents based on a network management application," Journal of communications and networks, Vol. 5, pp. 240-248, September 2003

[14] A. Fuggetta, G. P. Picco, and G. Vigna, "Understanding code mobility," IEEE Transactions on Software Engineering, vol. 24, pp. 342-361, 1998.

[15] Shivangi Katiyar and Devendra Prasad, "A Comprehensive Survey of Data Processing Approaches" International Journal of Computer Applications (IJCA), Volume 126, pp.38-45, September 2015. 
[16] Juby K Baby and P K Poonguzhali, "Energy Balanced Routing Method for In-Network Data Aggregation in Wireless Sensor Networks", IOSR Journal of Electronics and Communication Engineering (IOSR-JECE), Vol.9, Issue.3, pp. 5-14, June 2014.

[17] Mohammad Hossein Anisi, Abdul Hanan Abdullah and Shukor Abd Razak, "Energy-Efficient Data Collection in Wireless Sensor Networks", Science research journal, Vol.3, pp. 329-333, October 2011.

[18] Ali Norouzi, Faezeh Sadat Babamir and Zeynep Orman, "A Tree Based Data Aggregation Scheme for Wireless Sensor Networks Using GA", Scientific research journal, Vol. 4, pp. 191-196, August 2012.

[19] M. Francesco, D. Das, S.K. A and G. Anastasi, "Data Collection inWireless Sensor Networks with Mobile Elements: A Survey", 2011.

[20] Van Le, D. Oh, H.Yoon and S. HiCoDG, "A Hierarchical Data-Gathering Scheme Using Cooperative Multiple Mobile Elements", Sensors, pp. 24278-24304, 2014

[21] Tuhin Paul and Kevin Gordon Stanley, "Data Collection from Wireless Sensor Networks Using a Hybrid Mobile Agent-based Approach", In proceeding of the $39^{\text {th }}$ annual IEEE conference on Local Computer Networks, pp. 288295, 2014.

[22] Zheng Gengsheng and $\mathrm{Hu}$ Zhengbing, "Chain Routing based on Coordinates-oriented clustering strategy in
WSNs", In Proceeding of the IEEE conference on Computer Network and Multimedia Technology, pp. 1-4, January 2009.

[23] Onur Soysal and Murat Demirbas, "Data Spider: A Resilient Mobile Basestation Protocol for Efficient Data Collection in Wireless Sensor Networks", DCOSS, pp.393-408, 2010.

[24] Mohsin Raza Jafri, Nadeem Javaid, Akmal Javaid and Zahoor Ali Khan, "Maximizing the Lifetime of MultiChain PEGASIS Using Sink Mobility", World Applied Sciences Journal, pp. 1283-1289, 2013.

[25] Bartolom'e Rubio, Manuel D'iaz and Jos'e M. Troya, "Programming Approaches and Challenges for Wireless Sensor Networks" second IEEE international conference on systems and networks communications ICSNC, August 2007.

[26] Ozlem Durmaz Incel, Amitabha Ghosh, Bhaskar Krishnamachari, and Krishnakant Chintalapudi, "Fast Data Collection in Tree based Wireless Sensor Networks", IEEE Transactions on Mobile Computing, Vol. 11, No. 1, January 2012.

[27] Nakayama, H., Z. Fadlullah, N. Ansari and N. Kato, 2011. "A novel scheme for wsan sink mobility based on clustering and set packing techniques", IEEE Transactions on Automatic Control, Vol.56, No.20, pp. 2381-2389,

October

2011 